Pacific Journal of Mathematics

ON SATURATED FORMATIONS OF SOLVABLE LIE 


\title{
ON SATURATED FORMATIONS OF SOLVABLE LIE ALGEBRAS
}

\author{
ERNest L. STITZINGER
}

\begin{abstract}
The concepts of formations, $\mathscr{F}$-projectors and $\mathscr{F}$. normalizers have all been developed for solvable Lie algebras. In this note, for each saturated formation $\mathscr{F}$ of solvable Lie algebras, the class $\mathscr{T}(\mathscr{F})$ of solvable Lie algebras $L$ in which each $\mathscr{F}$-normalizer of $L$ is an $\mathscr{F}$-projector is considered. This is the natural generalization of the Lie algebra analogue to SC groups which were first investigated by $R$. Carter. It is shown that $\mathscr{T}(\mathscr{F})$ is a formation. Then some properties of $\mathscr{F}$-normalizers of $L \in \mathscr{T}(\mathscr{F})$ are considered.
\end{abstract}

All Lie algebras considered here are solvable and finite dimensional over a field $F$. $\mathscr{F}$ will always denote a saturated formation of solvable Lie algebras and $L$ will be a solvable Lie algebra. $N(L)$ is the nil-radical of $L$ and $\Phi(L)$ is the Frattini subalgebra of $L$. For definitions and properties of all these concepts see [3], [4], and [9]. For SC groups see [6].

We begin with a general lemma.

Lemma 1. Let $N$ be an ideal of $L$ and $D / N$ be an F -normalizer of $L / N$. Then there exists an $\mathscr{F}$-normalizer $E$ of $L$ such that $E+$ $N=D$.

Proof. Let $L$ be a minimal counterexample and we may assume that $N$ is a minimal ideal of $L$. If $D / N=L / N$, then any $\mathscr{F}$-normalizer of $L$ has the desired property, hence we may suppose that $D / N \subset L / N$. Suppose first that $N$ is $\mathscr{F}$-central in $L$. Let $N^{*} / N=$ $N(L / N)$ and $C=C_{L}(N)$. Then $N(L)=N^{*} \cap C$. Let $M / N$ be a maximal $\mathscr{F}$-critical subalgebra of $L / N$ such that $D / N$ is an $\mathscr{F}$ normalizer of $M / N$. Now either $M$ is $\mathscr{F}$-critical in $L$ or $M$ complements a chief factor of $L$ between $N^{*}$ and $N(L)$. In the first case, by induction, there exists an $\mathscr{F}$-normalizer $E$ of $M$ such that $E+$ $N=D$ and $E$ is also an $\mathscr{F}$-normalizer in $L$. In the second case, $L / C \in \mathscr{F}$ and $C+N^{*} / C$ is operator isomorphic to $N^{*} / N^{*} \cap C=N^{*} /$ $N(L)$. Hence each chief factor of $L$ between $N^{*}$ and $N(L)$ is $\mathscr{F}$. central which contradicts $M$ being $\mathscr{F}$-abnormal.

Now suppose that $N$ is $\mathscr{F}$-eccentric and assume $N \leqq \Phi(L)$. Let $M / N$ be as in the above paragraph. Again, by induction, there exists an $\mathscr{F}$-normalizer $E$ of $M$ such that $E+N=D$. But $N \leqq \Phi(L)$ yields that $M$ is $\mathscr{F}$-critical in $L$ using Theorem 2.5 of [4]. Hence $E$ is an 
$\mathscr{F}$-normalizer of $L$ and this case is completed.

Finally suppose that $N$ is $\mathscr{F}$-eccentric and assume $N \nsubseteq \Phi(L)$. Then $N$ is complemented by a maximal subalgebra $M$ which must be $\mathscr{F}$-critical in $L$. Now there must exist an $\mathscr{F}$-normalizer $E$ of $M$ such that $E+N=D$. Again $E$ must be an $\mathscr{F}$-normalizer of $L$ and the result is shown.

COROLlaRy. $\mathscr{T}(\mathscr{F})$ is closed under homomorphisms.

Proof. Let $N$ be a minimal ideal of $L, L \in \mathscr{T}(\mathscr{F})$. Let $D / N$ be an $\mathscr{F}$-normalizer of $L / N$. Then $D=E+N$ for some $\mathscr{F}$-normalizer of $L$. Now $E$ is an $\mathscr{F}$-projector of $L$ and $E+N / N=D / N$ is an $\mathscr{F}$-projector of $L / N$.

Lemma 2. If $L \in \mathscr{T}(\mathscr{F})$ and $C$ is an $\mathscr{F}$-projector of $L$, then $C$ is an $\mathscr{F}$-normalizer of $L$.

Proof. Let $N$ be a minimal ideal of $L . L / N \in \mathscr{T}(\mathscr{F})$ hence $C+N / N$ is an $\mathscr{F}$-normalizer of $L / N$ by induction. Hence $C+N=$ $D+N$ for some $\mathscr{F}$-normalizer $D$ of $L$. Now $D$ is also an $\mathscr{F}$-projector of $L$ and both $C$ and $D$ are $\mathscr{F}$-projectors of $C+N$. Then $C$ and $D$ are conjugate in $C+N$ by an inner automorphism of $C+N$ induced by an element of $N$ by Lemma 1.11 of [3]. Hence $D$ and $C$ are conjugate in $L$ and the result holds.

Note that $\mathscr{T}(\mathscr{F})$ contains a large class of Lie algebras. In fact by Theorem 3 of [9] we have

LEMMA 3. $\mathscr{N} \mathscr{F} \subseteq \mathscr{T}(\mathscr{F})$.

In order to obtain that $\mathscr{T}(\mathscr{F})$ is a formation, we record a characterization of $\mathscr{F}$-projectors which is completely analogous to a result in group theory due to Bauman [5]. Since the proofs carry over virtually unchanged, we omit them.

Definition. If $M$ is a subalgebra of $L$, then a series $0=L_{0} \subset$ $\cdots \subset L_{n}=L$ is called an $M$-series if $L_{i}$ is an ideal in $L_{i+1}$, if $M \cong$ $N_{L}\left(L_{i}\right)$ and if each $L_{i+1} / L_{i}$ is a nontrivial, irreducible $M$-factor of $L$.

THeOREM 1. If $C$ is an $\mathscr{F}$-projector of $L$ and $\left\{L_{i}\right\}, 0 \leqq i \leqq n$, is any $C$-series of $L$, then $C$ covers $L_{i} / L_{i-1}$ if and only if $C+L_{i}$ / $L_{i-1} \in \mathscr{F}$.

Proof. See proof of Theorem 1 of [5]. 
Theorem 2. If $\left\{L_{i}\right\}$ is a $C$-series of $L$ such that $C$ covers $L_{i} / L_{i-1}$ if and only if $C+L_{i} / L_{i-1} \in \mathscr{F}$, then $C$ is an $\mathscr{F}$-projector of $L$.

Proof. See proof of Theorem 2 of [5].

We intend to use these results in a slightly different form by means of

Lemma 4. Let $M$ be a subalgebra of $L, M \in \mathscr{F}$ and $H / K$ be a nontrivial, irreducible $M$-factor of $L$. Then $M+H / K \in \mathscr{F}$ if and only if the split extension of $H / K$ by $M / C_{M}(H / K)$ is in $\mathscr{F}$.

Proof. Since $M+H / H \in \mathscr{F}, M+H / K$ will be in $\mathscr{F}$ if and only if the minimal ideal $H / K$ of $M+H / K$ is $\mathscr{F}$-central in $M+H / K$; that is, if and only if the split extension of $H / K$ by $M+H / C_{M+H}(H / K)$ is in $\mathscr{F}$. But

$$
\begin{aligned}
M / C_{M}(H / K) & =M / M \cap C_{M+H}(H / K) \cong M+C_{M+H}(H / K) / C_{M+H}(H / K) \\
& =M+H / C_{M+I I}(H / K) .
\end{aligned}
$$

Now the corresponding split extensions of $H / K$ by $M+H / C_{M+I I}(H / K)$ and $H / K$ by $M / C_{M}(H / K)$ are isomorphic and the result holds.

\section{Theorem 3. $\mathscr{T}(\mathscr{F})$ is a formation.}

Proof. $\mathscr{T}(\mathscr{F})$ is closed under homomorphisms has been noted already. Hence let $N_{1}$ and $N_{2}$ be ideals of $L$ such that $L / N_{1}, L / N_{2} \in$ $\mathscr{T}(\mathscr{F})$. We may assume $N_{1} \cap N_{2}=0$ and show that $L \in \mathscr{T}(\mathscr{F})$. Let $D$ be an $\mathscr{F}$-normalizer of $L$. Then $D+N_{1} / N_{1}$ is an $\mathscr{F}$-normalizer of $L / N_{1}$, hence is an $\mathscr{F}$-projector of $L / N_{1}$ and the corresponding statement holds for $D+N_{2} / N_{2}$. Consider a $D$-series of $L$ which passes through $N_{1}$ and $N_{1}+N_{2}$. There is a $D$-series of $L$ which passes through $N_{2}$ and $N_{1}+N_{2}$ which is the same as the original $D$ series above $N_{1}+N_{2}$ and corresponds to the original $D$-series below $N_{1}+N_{2}$ in the natural way. In particular, a factor $H / K$ in the new $D$-series which is between $N_{2}$ and $N_{1}+N_{2}$ corresponds to $H \cap N_{1}$ l $K \cap N_{1}$ in the original $D$-series and we claim that $D$ covers (avoids) $H / K$ if and only if $D$ covers (avoids) $H \cap N_{1} / K \cap N_{1}$. For if $D$ avoids $H / K$, then $D \cap H \subseteq K$, hence $D \cap H \cap N_{1} \subseteq K \cap N_{1}$ and $D$ avoids $H \cap N_{1} / K \cap N_{1}$. Suppose that $D$ covers $H / K$. Then $H \leqq K+D$. In order to show that $D$ covers $H \cap N_{1} / K \cap N_{1}$ it is sufficient to show that $D+\left(K \cap N_{1}\right) \supseteqq H \cap N_{1}$. Since $H \subseteq K+D, D \subseteq N_{L}(K)$ and $H \cong$ $N_{1}+N_{2}$, it follows that $H \subseteq K+\left(D \cap\left(N_{1}+N_{2}\right)\right)$. Using the corollary on p. 241 of [9], $H \subseteq K+\left(\left(D \cap N_{1}\right)+\left(D \cap N_{2}\right)\right)=K+\left(D \cap N_{1}\right)$. Then, 
since $D \cap N_{1} \subset N_{L}(K)$ it follows that $H \cap N_{1} \subseteq\left(K+\left(D \cap N_{1}\right)\right) \cap N_{1} \subseteq$ $\left(K \cap N_{1}\right)+\left(D \cap N_{1}\right) \subseteq\left(K \cap N_{1}\right)+D$, hence $D$ covers $H \cap N_{1} / K \cap N_{1}$.

By Theorem 1 and Lemma 4, a factor $H / K$ above $N_{1}$ in the original $D$-series is covered by $D+N_{1} / N_{1}$ (hence $D$ ) if and only if the split extension of $H / K$ by $D+N_{1} / C_{D+N_{1}}(H / K)$ is in $\mathscr{F}$. That is, $H / K$ is covered by $D$ if and only if the split extension of $H / K$ by $D / C_{D}(H / K)$ is in $\mathscr{F}$. A similar statement holds above $N_{2}$. Every $D$-factor in the original series is operator isomorphic to a $D$-factor above $N_{1}$ or above $N_{2}$ and, using the result of the above paragraph, in the original $D$-series a factor $H / K$ is covered by $D$ if and only if the split extension of $H / K$ by $D / C_{D}(H / K)$ is in $\mathscr{F}$. Now by Lemma 4 and Theorem $2, D$ is an $\mathscr{F}$-projector of $L$ and $\mathscr{T}(\mathscr{F})$ is a formation.

The following example shows that $\mathscr{N} \mathscr{N} \subset \mathscr{T}(\mathscr{N})$ and that $\mathscr{T}(\mathscr{N})$ is not closed under taking ideals. It is a variant of an example on p. 52 of [7].

ExAmple. Let $F$ be a field of characteristic $p \geqq 2$ and let $A$ be a vector space over $F$ with basis $e_{0}, \cdots, e_{p-1}$. Define linear transformations $x, y, z$ on $A$ by

$$
\begin{aligned}
& x\left(e_{i}\right)=i e_{i} \\
& y\left(e_{i}\right)=e_{i+1}
\end{aligned}
$$

and

$$
z\left(e_{i}\right)=e_{i}
$$

$(\operatorname{subscripts} \bmod p)$. Then $[x, y]=x y-y x=y$ and $[x, z]=[y, z]=0$. Let $B$ be the three dimensional Lie algebra generated by $x, y, z$. Let $L$ be the semi-direct sum of $A$ and $B$ with the natural product. As on p. 53 of [7], $B$ acts irreducibly on $A$ so that $A$ is a minimal ideal of $L$. Evidently $A$ is self-centralizing in $L$, hence $A$ is the unique minimal ideal of $L$ and $N(L)=A$. Hence each $\mathscr{N}$-critical maximal subalgebra of $L$ complements $A$. Furthermore, $L$ is clearly of nilpotent length three.

Consider first any $\mathscr{N}$-normalizer $E$ of $L$ which is also an $\mathscr{N}$ normalizer of $B$. Such $\mathscr{N}$-normalizer exists since $B$ is a maximal $\mathscr{N}$-critical subalgebra of $L$. By the covering-avoidance property of $\mathscr{N}$-normalizers of $B, E=((z, x+\alpha y))$ where $\alpha \in F$. Now $B$ is of nilpotent length 2 , hence $E$ is a Cartan subalgebra of $B$. Now since $z \in E$, it is easily verified that $E$ is a Cartan subalgebra of $L$.

Now in general, each $\mathscr{N}$-normalizer of $L$ is an $\mathscr{N}$-normalizer of some $\mathscr{N}$-critical maximal subalgebra $M$ of $L$ and $M$ must complement $A$. But $L$ is of nilpotent length 3 and $L / A$ is of nilpotent length 2 , hence $M$ must be conjugate to $B$ by Theorem 8 of [8]. 
Consequently, any $\mathscr{N}$-normalizer of $L$ is a Cartan subalgebra of $L$ and $L \in \mathscr{T}(\mathscr{N})$.

Now the ideal $P=A+((x, y))$ of $L$ is not in $\mathscr{T}(\mathscr{N})$. For $((x)) \subset((x, y)) \subset P$ is a maximal $\mathscr{N}$-critical chain of $P$, hence $((x))$ is an $\mathscr{N}$-normalizer of $P$. However, the normalizer of $((x))$ in $P$ is $\left(\left(x, e_{0}\right)\right)$. Hence $L \notin \mathscr{T}(\mathscr{N})$.

We recall that each $\mathscr{F}$-normalizer is contained in an $\mathscr{F}$-projector (Theorem 6 of [9]). However, the usual converse result, namely each $\mathscr{F}$-projector contains an $\mathscr{F}$-normalizer has not been obtained, even for $\mathscr{N} \mathscr{N} \mathscr{F}$-Lie algebras. We now show that this result holds if $L \in \mathscr{N} \mathscr{T}(\mathscr{F})$. First we record the following result which is needed.

THEOREM 4. Let $L \in \mathscr{N} \mathscr{T}(\mathscr{F})$. Then each $\mathscr{F}$-normalizer of $L$ is contained in a unique $\mathscr{F}$-projector of $L$.

Proof. Same as the proof of Theorem 9 of [9].

Theorem 5. Let $L \in \mathscr{N} \mathscr{T}(\mathscr{F})$. Then each $\mathscr{F}$-projector of $L$ contains an $\mathscr{F}$-normalizer of $L$.

Proof. Let $N$ be a minimal ideal of $L$ and let $C$ be an $\mathscr{F}$ projector of $L$. Then $C+N / N$ is an $\mathscr{F}$-projector of $L / N$ and $C+$ $N / N$ contains an $\mathscr{F}$-normalizer $D / N$ of $L / N$ by induction. Let $T=$ $C+N$ and let $F$ be an $\mathscr{F}$-normalizer of $L$ such that $F+N=D \subseteq T$. Then $F$ is contained in an $\mathscr{F}$-projector $G$ of $L$ and $D / N \leqq G+N / N$. Hence $G+N=C+N$ by Theorem 4 and $G$ and $C$ are $\mathscr{F}$-projectors of $T$. By Lemma 1.11 of [3], $G$ and $C$ are conjugate in $T$ by an inner automorphism of $T$ induced by an element of $N$. Hence $G$ and $C$ are conjugate in $L$ and the result holds.

$\mathscr{F}$-normalizers have the covering-avoidance property but the converse is not true in general. However, if $L \in \mathscr{T}(\mathscr{F})$, then the converse is true.

Theorem 6. Let $L \in \mathscr{T}(\mathscr{F})$. If $D$ is a subalgebra of $L$ which covers the $\mathscr{F}$-central chief factors of $L$ and avoids the $\mathscr{F}$-eccentric chief factors of $L$, then $D$ is an $\mathscr{F}$-normalizer of $L$.

Proof. Let $N$ be a minimal ideal of $L$. Then $D+N / N$ has the covering-avoidance property in $L / N \in \mathscr{T}(\mathscr{F})$. By induction, $D+N / N$ is an $\mathscr{F}$-normalizer of $L / N$ and $D+N=E+N=T$ for some $\mathscr{F}$-normalizer $E$ of $L$. Since $L \in \mathscr{T}(\mathscr{F}), E$ is an $\mathscr{F}$-projector of $L$ and then also of $T$. If $N$ is $\mathscr{F}$-central in $L$, then $N \subseteq D$ and $N \subseteq E$, hence $D=E$. Suppose $N$ is $\mathscr{F}$-eccentric. Then $D \cap N=0=E \cap N$. Now $T \in \mathscr{N} \mathscr{F}$, hence $E$ is an $\mathscr{F}$-normalizer of $T$ by Theorem 3 of [9]. Furthermore, in a given chief series of $T$ passing through $N, \mathbb{F}$ 
covers all chief factors above $N$ and avoids all chief factors below $N$ and the same is true for $D$. Since $E$ is an $\mathscr{F}$-normalizer of $T$, each chief factor below $N$ must be $\mathscr{F}$-eccentric and each chief factor above $N$ must be $\mathscr{F}$-central. Hence, by Theorem 4 of [9], $D$ must be an $\mathscr{F}$-normalizer of $T$. By Theorem 3 of [9], $D$ must also be an $\mathscr{F}$-projector of $T$. Now $D$ and $E$ are conjugate in $T$ (hence in $L$ ) by an inner automorphism induced by an element of $N$. Hence $D$ is an $\mathscr{F}$-normalizer of $L$.

Henceforth we shall be concerned with the case $\mathscr{F}=\mathscr{N}$. Here we have the following stronger form of Theorem 4 .

Theorem 7. Let $L \in \mathscr{N} \mathscr{T}(\mathscr{N})$ and $D$ be an $\mathscr{N}$-normalizer of $L$. Then there exists a Cartan subalgebra $C$ of $L$ which contains every subalgebra $H$ of $L$ in which $D$ is subinvariant. In particular, $D$ is contained in a unique Cartan subalgebra of $L . \quad C$ is the Fitting null component of $D$ acting on $L$.

Proof. $D+N(L) / N(L)$ is subinvariant in $H+N(L) / N(L)$ and $D+N(L) / N(L)$ is an $\mathscr{N}$-normalizer of $L / N(L) \in \mathscr{T}(\mathscr{N})$. Hence $D+N(L) / N(L)=H+N(L) / N(L)$ is a Cartan subalgebra of $L / N(L)$. Let $T=D+N(L)=H+N(L)$ and let $S$ be the Fitting null component of $D$ acting on $T$. Evidently $N_{T}(S)=S$ and $H \subseteq S$. Furthermore, $S=S \cap T=S \cap(D+N(L))=D+(S \cap N(L))$. Each element of $D$ induces a nilpotent derivation on $S$ and $S \cap N(L)$ is a nilpotent ideal of $S$. Then, using Engel's theorem, $S$ is nilpotent. Hence $S$ is a Cartan subalgebra of $T$ and also of $L$ by Lemma 1.8 of [3]. If $K$ is another Cartan subalgebra of $L$ containing $D$, then $D$ is subinvariant in $K$, hence $K=S$. The last past of the theorem follows from the next lemma.

Lemma 5. Let $L$ be a solvable Lie algebra and $D$ be a nilpotent subalgebra of $L$. Let $F$ be the Fitting null component of $D$ acting on $L$. Then $D$ is subinvariant in $F$.

Proof. We may suppose that $F=L$. Let $A$ be a minimal ideal of $L$. Now in $D+A, A$ is an abelian ideal and each element of $D$ induces a nilpotent derivation of $D+A$. Hence, using Engel's theorem, $D+A$ is nilpotent and $D$ is subinvariant in $D+A$. But $D+A / A$ satisfies the conditions in $L / A$, hence $D+A / A$ is subinvariant in $L / A$ by induction. Therefore, $D$ is subinvariant in $L$.

For Lie algebras of nilpotent length three, a result somewhat stronger than Theorem 7 holds. The proof is the same as the proof of Theorem 7, using Theorem 1 of [8] instead of the defining property of $\mathscr{T}(\mathscr{N})$, and may be omitted. 
THEOREM 8. Let $L$ be of nilpotent length three (or less) and let $D$ be a nilpotent subalgebra of $L$ which can be joined to $L$ by a maximal chain of subalgebras, each self-normalizing in the next. Then there exists a Cartan subalgebra $C$ of $L$ which contains every subalgebra $H$ of $L$ in which $D$ is subinvariant. In particular, $D$ is contained in a unique Cartan subalgebra $C$ of $L$ and $C$ is the Fitting null component of $D$ acting on $L$.

We may use this to find a Lie algebra analogue to Theorem 10 of [2].

THEOREM 9. Let $M$ be a self-normalizing maximal subalgebra of L. Suppose that $L$ is of nilpotent length three. Then each Cartan subalgebra of $M$ is of the form $M \cap C$ for some Cartan subalgebra $C$ of $L$.

Proof. Let $D$ be a Cartan subalgebra of $M$. Then $D$ is contained in a Cartan subalgebra $C$ of $L$ by Theorem 8 and Lemma 1 of [8]. Now $M \cap C$ is nilpotent and $D$ is a Cartan subalgebra of $M \cap C$. Hence $D=M \cap C$.

The final result is of a slightly different nature. We consider the following: If an $\mathscr{N}$-normalizer $D$ of $L$ is contained in the selfnormalizing maximal subalgebra $M$ of $L$, then is $D$ contained in an $\mathscr{N}$-normalizer of $M$. The analogous question for finite groups is answered negatively in [1]. The Lie algebra case also has a negative answer as in shown in the following result. The second part of this example is also an analogue to the example of [1].

THEOREM 10. There exists a solvable Lie algebra $L \in \mathscr{N} \mathscr{N} \mathscr{N}$ which has an $\mathscr{N}$-normalizer $D$, ideal $A$ and maximal subalgebra $M$ containing $D$ such that

(1) $D$ is not contained in an $\mathscr{N}$-normalizer of $M$

(2) $N_{L / A}(D+A / A) \supset N_{L}(D)+A / A$.

Proof. This example is also a variant of an example found on p. 52 of [7]. Let $F$ be a field of characteristic $p>2$. Let $A$ be the Lie algebra over $F$ with basis $a_{0}, a_{1}, \cdots, a_{p-1}, b, c_{0}, c_{1}, \cdots, c_{p-1}$ and products $\left[a_{i}, b\right]=c_{i}$ for $i=0, \cdots, p-1$ and all other products of basis elements equal to 0 . Define linear transformations $x, y$ on $A$ such that

$$
\begin{array}{rlrl}
x\left(a_{i}\right) & =a_{i+1} & y\left(a_{i}\right) & =i a_{i} \\
x(b) & =0 & y(b) & =0 \\
x\left(c_{i}\right) & =c_{i+1} & y\left(c_{i}\right) & =i c_{i}
\end{array}
$$


(everything $\bmod p$ ). Then $x$ and $y$ are derivations of $A$ and $[y, x]=x$. Let $B$ be the 2-dimensional Lie algebra generated by $x$ and $y$ and let $L$ be the semi-direct sum of $A$ and $B$ with the natural product.

Let $R=\left(\left(c_{0}, \cdots, c_{p-1}\right)\right)$ and $S=\left(\left(c_{0}, \cdots, c_{p-1}, b\right)\right)$. The same argument used in [7] shows that $R$ and $A / S$ are $\mathscr{N}$-eccentric chief factors of $L$ and $S / R$ is clearly and $\mathscr{N}$-central chief factor of $L$. Let $M=$ $\left(\left(x, y, b, c_{0}, \cdots, c_{p-1}\right)\right), M_{1}=((x, y, b))$ and $M_{2}=((y, b))$. Each of these is a maximal $\mathscr{N}$-critical subalgebra of the preceding and $M$ is maximal, $\mathscr{N}$-critical in $L$. Now $\exp a_{0}$ is an automorphism of $L$ since char $F \neq 2$. Then $C=M_{2}^{\exp a_{0}}=\left(\left(y, b+c_{0}\right)\right) \subseteq M$ and $D$ is an $\mathscr{N}$ normalizer of $L$.

Now the $\mathscr{N}$-normalizers of $M$ have dimension 2 by the coveringavoidance property of $\mathscr{N}$-normalizers, hence, if $D$ is contained in an $\mathscr{N}$-normalizer of $M$, then it is one of them. If this is the case, then, since $b \in Z(M), b \in D$ and $\operatorname{dim} D>2$, a contradiction.

For the second part, note that

$$
N_{L / R}\left(M_{2}+R / R\right)=\left(\left(y+R, b+R, a_{0}+R\right)\right) .
$$

However, an element of the form $\alpha a_{0}+t, \alpha \in F, t \in R$ is not in $N_{L}\left(M_{2}\right)$ unless $\alpha=0$, since $\left[b, \alpha a_{0}+t\right]=-\alpha c_{0}$. Hence

$$
N_{L}\left(M_{2}\right)+R / R \subset N_{L / R}\left(M_{2}+R / R\right) \text {. }
$$

\section{REFERENCES}

1. J. L. Alperin, Normalizers of system normalizers, Trans. Amer. Math. Soc., 113 (1964), 181-188.

2. - System normalizers and Carter subgroups, J. Algebra, 1 (1964), 355-366.

3. D. W. Barnes and H. M. Gastineau Hills, On the theory of solvable Lie algebras, Math. Z., 106 (1968), 343-354.

4. D. W. Barnes and M. L. Newell, Some theorems on saturated homomorphs of solvable Lie algebras, Math. Z., 115 (1970), 179-187.

5. S. Bauman, $A$ note on cover and avoidance properties in solvable groups, Proc. Amer. Math. Soc., 21 (1969), 173-174.

6. B. Huppert, Endliche Gruppen I, Springer-Verlag, Berlin-Heidelberg-New York, 1967.

7. N. Jacobson, Lie Algebras, Interscience, New York-London-Sydney, 1966.

8. E. L. Stitzinger, Theorems on Cartan subalgebras like some Carter subgroups, Trans. Amer. Math. Soc., 159 (1971), 307-315.

9. Covering-avoidance for saturated formations of solvable Lie algebras, Math. Z., 124 (1972), 237-249.

Received June 8, 1972.

North Carolina State University 


\section{PACIFIC JOURNAL OF MATHEMATICS}

\section{EDITORS}

D. Gilbarg AND J. MILGRAM

Stanford University

Stanford, California 94305

\section{R. A. Beaumont}

University of Washington

Seattle, Washington 98105

\section{J. DUGUNDJI*}

Department of Mathematics University of Southern California Los Angeles, California 90007

RICHARD ARENS

University of California Los Angeles, California 90024

\section{ASSOCIATE EDITORS}
E. F. BECKENBACH
B. H. NEUMANN
F. WOLF
K. YoshidA

\section{SUPPORTING INSTITUTIONS}

\author{
UNIVERSITY OF BRITISH COLUMBIA \\ CALIFORNIA INSTITUTE OF TECHNOLOGY \\ UNIVERSITY OF CALIFORNIA \\ MONTANA STATE UNIVERSITY \\ UNIVERSITY OF NEVADA \\ NEW MEXICO STATE UNIVERSITY \\ OREGON STATE UNIVERSITY \\ UNIVERSITY OF OREGON \\ OSAKA UNIVERSITY
}

\author{
UNIVERSITY OF SOUTHERN CALIFORNIA \\ STANFORD UNIVERSITY \\ UNIVERSITY OF TOKYO \\ UNIVERSITY OF UTAH \\ WASHINGTON STATE UNIVERSITY \\ UNIVERSITY OF WASHINGTON \\ AMERICAN MATHEMATICAL SOCIETY \\ NAVAL WEAPONS CENTER
}

The Supporting Institutions listed above contribute to the cost of publication of this Journal, but they are not owners or publishers and have no responsibility for its content or policies.

Mathematical papers intended for publication in the Pacific Journal of Mathematics should be in typed form or offset-reproduced, (not dittoed), double spaced with large margins. Underline Greek letters in red, German in green, and script in blue. The first paragraph or two must be capable of being used separately as a synopsis of the entire paper. Items of the bibliography should not be cited there unless absolutely necessary, in which case they must be identified by author and Journal, rather than by item number. Manuscripts, in duplicate if possible, may be sent to any one of the four editors. Please classify according to the scheme of Math. Rev. Index to Vol. 39. All other communications to the editors should be addressed to the managing editor, Richard Arens, University of California, Los Angeles, California, 90024.

50 reprints are provided free for each article; additional copies may be obtained at cost in multiples of 50 .

The Pacific Journal of Mathematics is issued monthly as of January 1966. Regular subscription rate: $\$ 48.00$ a year (6 Vols., 12 issues). Special rate: $\$ 24.00$ a year to individual members of supporting institutions.

Subscriptions, orders for back numbers, and changes of address should be sent to Pacific Journal of Mathematics, 103 Highland Boulevard, Berkeley, California, 94708.

PUBLISHED BY PACIFIC JOURNAL OF MATHEMATICS, A NON-PROFIT CORPORATION

Printed at Kokusai Bunken Insatsusha (International Academic Printing Co., Ltd.), 270, 3-chome Totsuka-cho, Shinjuku-ku, Tokyo 160, Japan.

* C. DePrima will replace J. Dugundji until August 1974.

Copyright (C) 1973 by

Pacific Journal of Mathematics

All Rights Reserved 


\section{Pacific Journal of Mathematics}

\section{Vol. 47, No. $2 \quad$ February, 1973}

David Parham Bellamy, Composants of Hausdorff indecomposable continua; a mapping approach ........................ 303

Colin Bennett, A Hausdorff-Young theorem for rearrangement-invariant spaces ...........................................

Roger Daniel Bleier and Paul F. Conrad, The lattice of closed ideals and $a^{*}$-extensions of an abelian l-group ...

Ronald Elroy Bruck, Jr., Nonexpansive projections on subsets of Banach

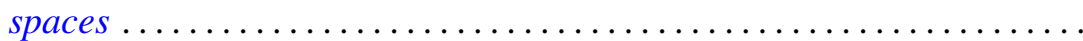

Robert C. Busby, Centralizers of twisted group algebras ............. 357

M. J. Canfell, Dimension theory in zero-set spaces ................ 393

John Dauns, One sided prime ideals ........................ 401

Charles F. Dunkl, Structure hypergroups for measure algebras . . . . . . . . . 413

Ronald Francis Gariepy, Geometric properties of Sobolev mappings ...... 427

Ralph Allen Gellar and Lavon Barry Page, A new look at some familiar spaces of intertwining operators ...........................

Dennis Michael Girard, The behavior of the norm of an automorphism of the

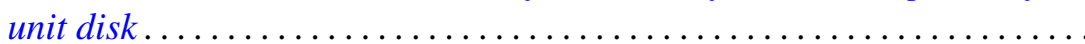

George Rudolph Gordh, Jr., Terminal subcontinua of hereditarily

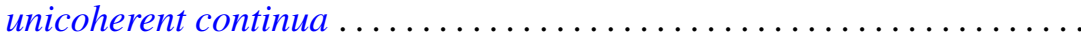

Joe Alston Guthrie, Mapping spaces and cs-networks. .

Neil Hindman, The product of $F$-spaces with $P$-spaces . 473

M. A. Labbé and John Wolfe, Isomorphic classes of the spaces $C_{\sigma}(S)$

Ernest A. Michael, On k-spaces, $k_{R}$-spaces and $k(X) \ldots$

Donald Steven Passman, Primitive group rings .

C. P. L. Rhodes, A note on primary decompositions of a pseudovaluation ...

Muril Lynn Robertson, A class of generalized functional differential equations

Ruth Silverman, Decomposition of plane convex sets. $I$.

Ernest Lester Stitzinger, On saturated formations of solvable Lie algebras................................

B. Andreas Troesch, Sloshing frequencies in a half-space by Kelvin inversion ...

L. E. Ward, Fixed point sets .

Michael John Westwater, Hilbert transforms, and a problem in scattering

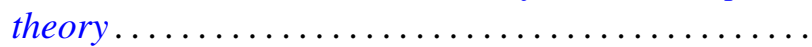

Misha Zafran, On the spectra of multipliers... 